\title{
Effect of Hot Rolling Parameters on Texture and Anisotropy of X65MO Offshore Pipeline Steel
}

\author{
Tao Niu* \\ Shougang Research Institute of Technology, \\ Shougang Group, Beijing, 100043, China; \\ E-mail: johnniu@126.com \\ Xinlang Wu \\ Shougang Qian'an Iron \&Steel Co.Ltd, \\ Qian'an, Hebei Province, 064404, China; \\ E-mail: wuxinlang2160@sgqg.com
}

\section{Chen $\mathrm{Yu}$}

Shougang Qian'an Iron \&Steel Co.Ltd, Qian'an, Hebei Province, 064404, China; E-mail:yuchen@sgqg.com

\begin{abstract}
In this study, optical microscope (OM), tensile test, X-ray diffraction (XRD) method were used to investigate the effect of hot rolling parameters on texture and tensile properties of X65MO offshore pipeline steel. Result of microstructure observation reveals that the anisotropy of mechanical properties of X65MO submarine pipeline steel is not due to the different growing direction of grains, but to the different crystal orientations - texture. And the texture mainly consists of near $\{112\}<110>,\{111\}<112>$ and $\{001\}<110>$ for hot rolled X65MO offshore pipeline steel, which depends on the texture of austenite and its revolution during phase transformation process. Increasing the finishing rolling temperature can reduce the density of $\{112\}<110\rangle$ texture dramatically and correspondingly reduce the anisotropy, while higher deformation will increase $\{112\}<110>$ component and intensify the anisotropy of strength. Reason analysis suggests that the key point is to reduce the copper texture and increase the others instead. Optimized finishing rolling temperature and transfer bar thickness were recommended based on the investigation.
\end{abstract}

Keywords-X65MO; offshore pipeline steel; texture anisotropy; hot rolling; parameters

\author{
Chenggang An \\ Shougang Research Institute of Technology, \\ Shougang Group, Beijing, 100043, China; \\ E-mail: anchegnggang@shougang.com.cn \\ Caixia Zhang \\ Shougang Qian'an Iron \&Steel Co.Ltd, Qian'an, \\ Hebei Province, 064404, China; \\ E-mail: zhangcaixia@sgqg.com
}

\section{INTRODUCTION}

Submarine steel pipeline is one of the most important way to transport natural gas from ocean platform to land. Comparing with terrestrial pipelines, offshore pipelines are either placed directly on the seabed or suspended in the water. Additional structural tensile stress is generated by changes in the submarine topography, ocean current and geological factors, etc. [1]. Therefore, performance requirement of high strength, high deformation ability, good toughness and weld ability is getting more and more complex.

As is well known, tensile property anisotropy has a significant influence on the deformation ability of line pipe [2]. In the past research, relationship between texture and anisotropy has been investigated and anisotropic models has been built to predict the forming limit strains based on texture and dislocation structure [3 4].

Meanwhile, researchers have put forward a lot of experiments to reveal the effects of texture on fracture behaviour of pipeline steels, for example, Charpy toughness of X80 pipeline steel [5 6], but 
there are still few investigations for the tensile properties, especially for X65MO submarine pipeline steel.

The purpose of the present work is to study how the texture influences anisotropy of tensile performance for submarine pipeline steel, especially to provide the production recommendations to improve it by adjusting hot rolling parameters.

\section{MATERIALS AND METHODS}

$\mathrm{X} 65 \mathrm{MO}$ used in this work were sampled from submarine pipeline steel hot rolled on $2250 \mathrm{~mm}$ hot continuous rolling line, and the main chemical compositions are shown in Table 1. After reheated to $1150 \sim 1250^{\circ} \mathrm{C}$, the slab was hot rolled using a twostage themo-mechanical process and laminar cooling. The thickness of slab is about $230 \mathrm{~mm}$ and the final coil is about $8.7 \mathrm{~mm}$.

TABLE 1: CHEMICAL COMPOSITION OF X65MO (WT, \%)

\begin{tabular}{cccccccc}
\hline $\mathrm{C}$ & $\mathrm{Si}$ & $\mathrm{Mn}$ & $\mathrm{P}$ & $\mathrm{S}$ & $\mathrm{Others}$ & $\mathrm{Ceq}$ \\
\cline { 2 - 7 } $0.05 \sim 0.08$ & $0.10 \sim 0.30$ & $1.40 \sim 1.60$ & $\leq 0.010$ & $\leq 0.0020$ & $\mathrm{Nb}, \mathrm{V}, \mathrm{Ti}, \mathrm{Cr}$ & 0.35 & 0.15 \\
\hline
\end{tabular}

In order to investigate the influence of hot rolling parameters on texture and properties, different intermediate slab thickness $(42 / 46 \mathrm{~mm})$ and finishing rolling temperatures $\left(900 / 860 / 820^{\circ} \mathrm{C}\right)$ were designed. After production, samples were taken from the coil to test the tensile properties in different directions, including transverse, $30^{\circ}$ to longitudinal, $60^{\circ}$ to longitudinal and rolling direction. Meanwhile, the macro texture was measured by utilizing XRD technology to investigate the texture changes under different rolling conditions.

\section{RESULTS ANALYSIS AND DISCUSSION}

\section{A. Microstructure in Transverse and Rolling} Direction

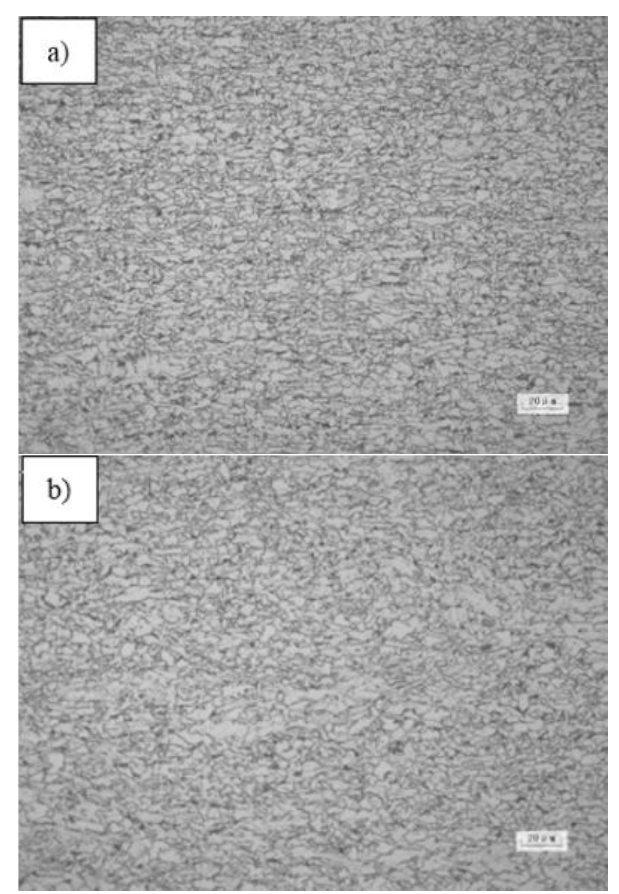

Figure 1. Microstructures obtained in different directions a) transverse; b) longitudinal
First of all, microstructures obtained in transverse and rolling direction have been observed for $\mathrm{X} 65 \mathrm{MO}$ submarine pipeline steel (Figure 1). The microstructure of X65MO mainly consists of homogenous refined polygonal ferrite and some amount of perlite, and there is no obvious characteristic of directional alignment for the ferrite grains. The reason might be that for pre-eutectoid ferrite, the grain tends to grow to be equi-axed since the dynamic condition is sufficient at higher temperature. Therefore, it can be revealed that the anisotropy of mechanical properties is not due to the different growing direction of grains, but to the different crystal orientations - texture.

The anisotropy of tensile stress caused by texture can be explained and analysed by Taylor factor. Generally, shearing stress on different glide plane can be considered the same, so the tensile stress mainly depends on the different Taylor factors. In Chen wen's study [7], it has been revealed that the Taylor factor variation of $\{112\}<110>$ texture well matched with the anisotropy of tensile properties. Therefore, it is the most effective way to reduce the anisotropy of pipeline steel by the reduction of $\{112\}<110>$ texture component. 
B. Effect of Finishing Rolling Temperature on Texture and Property
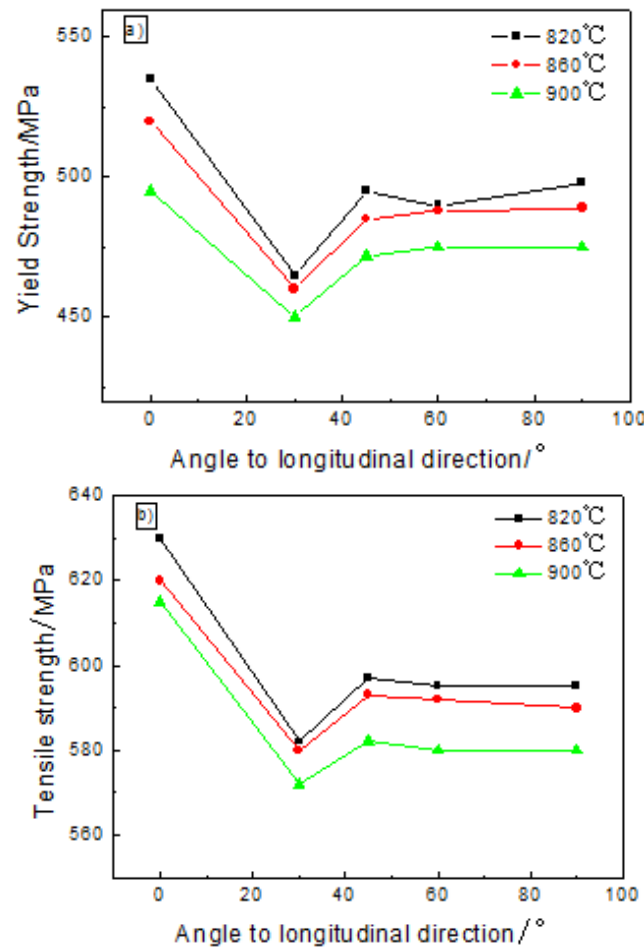

Figure 2. Tensile properties obtained with different finishing rolling temperatures a) yield strength; b) tensile strength

Figure 2 shows the yield and tensile strength along different directions under different finishing rolling temperatures, including $900^{\circ} \mathrm{C}, 860^{\circ} \mathrm{C}$, and $820^{\circ} \mathrm{C}$. Normally, the yield strength reaches the highest in transverse direction and the lowest in $30^{\circ}$ to longitudinal direction. Both the yield strength and tensile strength decrease with the increment of finishing rolling temperatures. It can be easily explained that the grain refinement of austenite reduces on some level because of the deformation has been put forward at higher temperature. And in condition of lower deformation accumulation, nucleation rate of ferrite also drops and the finial grain of ferrite gets coarse. However, the anisotropy of strength has been improved with the increment of finishing rolling temperatures. For example, when the finishing rolling temperature increases from $820^{\circ} \mathrm{C}$ to $900^{\circ} \mathrm{C}$, the difference between max. and min. of yield strength drops from $70 \mathrm{MPa}$ to $45 \mathrm{MPa}$.
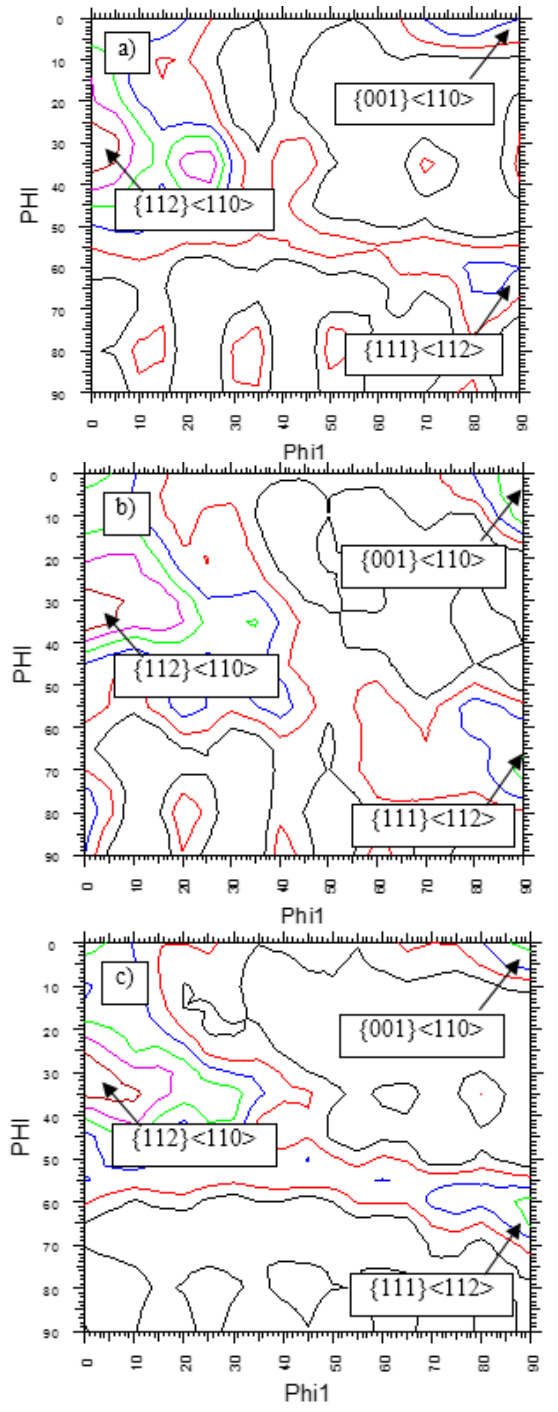

Figure 3. ODF section view obtained with different finishing rolling temperatures a) $900^{\circ} \mathrm{C}$; b) $860^{\circ} \mathrm{C}$; c) $820^{\circ} \mathrm{C}$

Figure 3 shows the orientation distribution function (ODF) results obtained with different finishing rolling temperatures in non - recrystrallization region, $900^{\circ} \mathrm{C} ; 860^{\circ} \mathrm{C}$ and $820^{\circ} \mathrm{C}$. It can be concluded that the texture of hot rolled X65MO steel mainly consists of near $\{112\}\langle 110\rangle, \quad\{111\}<112\rangle$ and $\{001\}<110\rangle$, and $\{001\}<110\rangle$ is very weak with finishing rolling temperature of $900^{\circ} \mathrm{C} .\{112\}<110>$ is the strongest texture, while near $\{112\}<110>$ occupies the second place. 

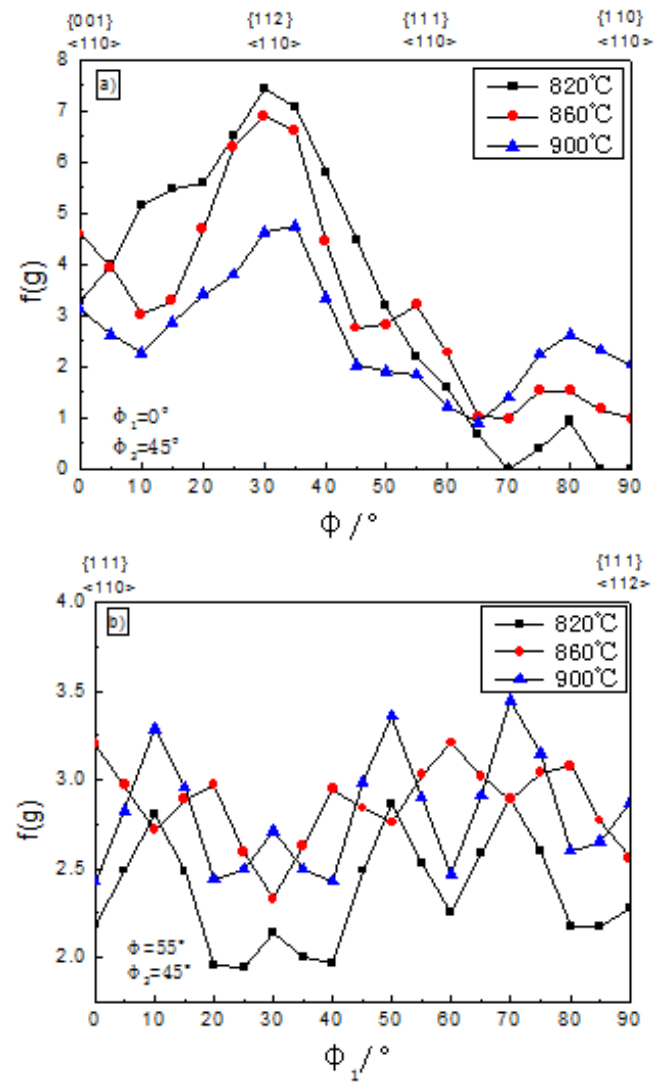

Figure 4. Orientation densities along $\alpha$ and $\gamma$ fibers with different hot rolling temperatures a) $\alpha$; b) $\gamma$

Figure 4 shows the orientation densities along $\alpha$ and $\gamma$ fibers with different hot rolling temperatures. When the finishing rolling temperature decreases from $900^{\circ} \mathrm{C}$ to $820^{\circ} \mathrm{C}$, the components of all the textures tends to increase in different degrees. Comparatively, the density of $\{112\}<110\rangle$ texture increases the most, which helps to explain why the yield strength anisotropy decreases with the increment of finishing rolling temperature.

Many previous studies have investigated the texture of austenite and its revolution during phase transformation process for pipeline steels. As has been revealed, the $\{112\}<110>$ texture in room temperature microstructure mainly originates in Copper texture of austenite (rolling texture $\{112\}<111>$ ) [8]. When the rolling temperature decreases, copper texture tends to transform to be $\{112\}\langle 110\rangle$. On the other hand, some part of $\{112\}<110>$ texture transforms from Goss texture $(\{110\}<001>)$, which can also transforms to $\{111\}<110>$ texture partially, especially when the rolling temperature is higher [9]. Therefore, it is the most efficient way to control the strength anisotropy by reducing the component proportion of copper texture in the deformed austenite, as well as to reduce the transformation possibility of Goss texture. According to the investigation results, higher deformation temperature can change the texture component of face centered cubic material, increase the component of Brass and $\mathrm{S}$ type texture, and correspondingly reduce that of Copper texture [8-9]. Therefore, with the increment of finishing rolling temperature, the $\{112\}<110>$ texture component becomes lower and the anisotropy problem gets improved.

Although the anisotropy of strength can be weakened by higher finishing rolling temperature, it cannot be increased for too high, which can cause the obvious reduction of strength for the pipeline steel, as is shown in Figure 2. Therefore, combined to the strength requirement lower limit of steel, $860^{\circ} \mathrm{C}$ is recommended for $\mathrm{X} 65 \mathrm{MO}$ offshore pipeline steel production.

\section{Effect of Intermediate Slab Thickness on} Texture and property
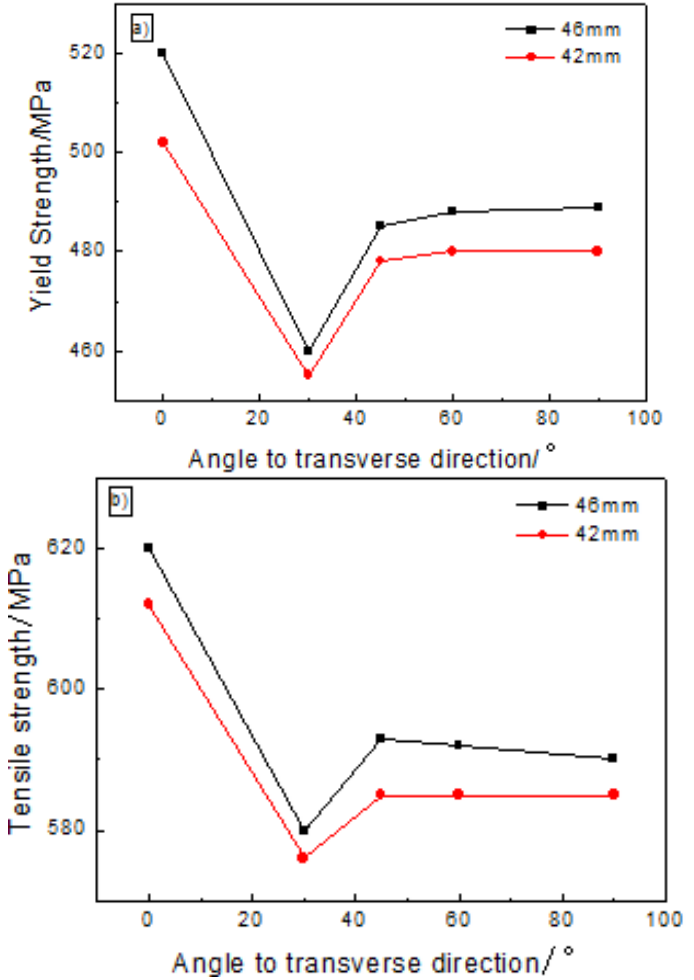

Figure 5. Tensile properties obtained with different transfer bar thickness a) yield strength; b) tensile strength

Yield and tensile strength results along different directions obtained under different transfer bar thickness are shown in Figure 5. Similar to effect of finishing rolling temperatures increment, yield and tensile strength both decrease with the decrement of transfer bar thickness because of the less grain refinement effect with less deformation accumulation in the non- recrystallization region. Meanwhile, the yield strength anisotropy also become weakened with the decrement of transfer bar thickness. 

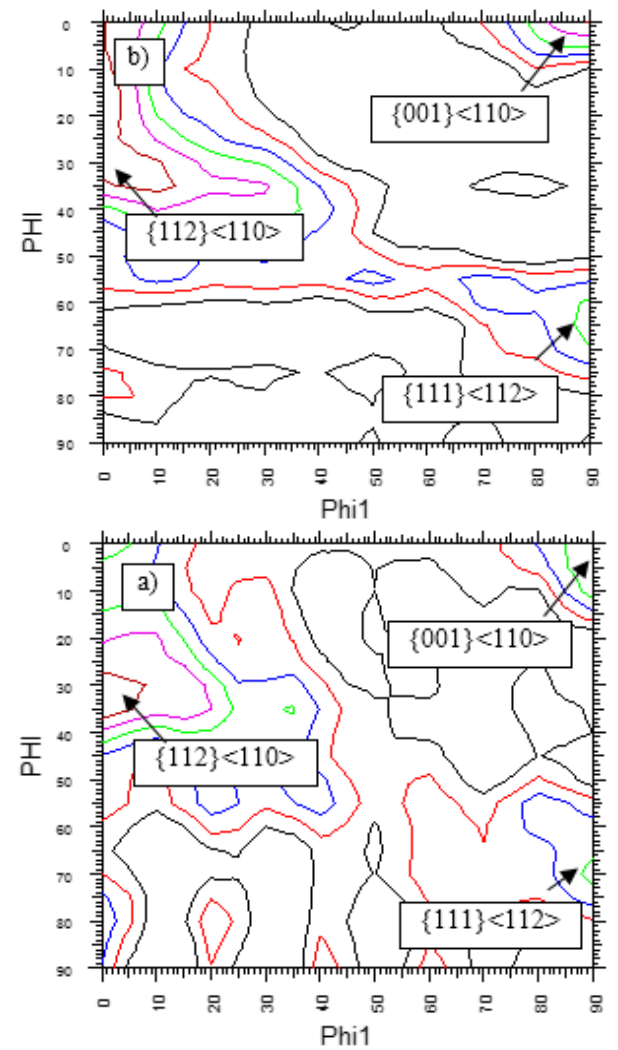

Figure 6. ODF section view obtained with different transfer bar thickness a) $42 \mathrm{~mm}$; b) $46 \mathrm{~mm}$
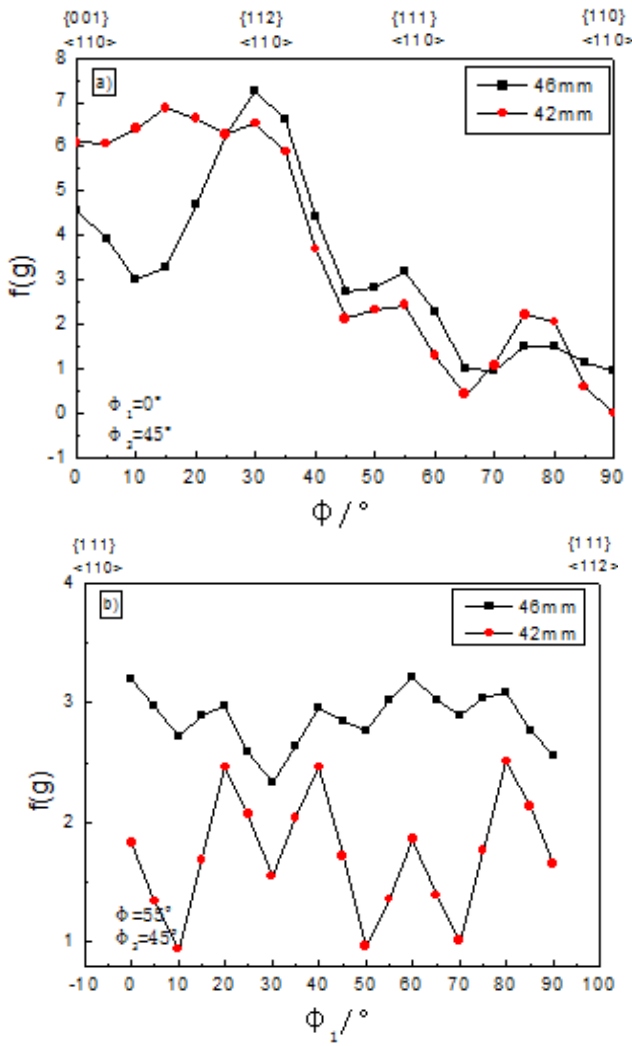

Figure 7. Orientation densities along $\alpha$ and $\gamma$ fibers with different transfer bar thickness a) $\alpha$; b) $\gamma$

Figure 7 shows the orientation densities variation along $\alpha$ and $\gamma$ fibers with different transfer bar thickness. Together with the ODF shown in Figure 6, it can be found that the component of near $\{112\}<110\rangle$ texture increases obviously with the increment of transfer bar thickness, which reflects the correspondence with variation of strength anisotropy. As is well known, for face centered cubic material, all the texture components will increase under higher deformation, especially Copper, Brass and $\mathrm{S}$ textures. Therefore, with the increment of deformation, more Copper texture can provide higher density of near $\{112\}<110\rangle$ component. The same situation occurs for Goss texture too, which causes the further increment of $\{112\}<110\rangle$ component [10]. From this angle, properly reducing the transfer bar thickness, and the deformation in non recrystallization region contributes to reduce the anisotropy of strength.

\section{IV.CONCLUSIONS}

Microstructure observation reveals that the anisotropy of mechanical properties of X65MO submarine pipeline steel is not due to the different growing direction of grains, but to the differ-ent crystal orientations - texture. And the texture mainly consists of near $\{112\}\langle 110\rangle,\{111\}\langle 112\rangle$ and $\{001\}<110\rangle$.

When the finishing rolling temperature decreases from $900^{\circ} \mathrm{C}$ to $820^{\circ} \mathrm{C}$, the density of $\{112\}<110>$ texture increases most dramatically, as well as the anisotropy. Considering the strength requirement lower limit of steel, $860^{\circ} \mathrm{C}$ is recommended for $\mathrm{X} 65 \mathrm{MO}$ offshore pipeline steel production.

Texture analysis suggests that higher deformation will increase $\{112\}\langle 110\rangle$ component on some level. Therefore, properly reducing the transfer bar thickness, and the deformation in non recrystallization region contributes to reduce the anisotropy of strength.

\section{REFERENCES}

References are cited in the text just by square brackets [1]. Two or more references at a time may be put in one set of brackets $[3,4]$. The references are to be numbered in the order in which they are cited in the text and are to be listed at the end of the contribution under a heading References, see our example below.

[1] F. L. Sun, S. Ren, Z Li, Z.Y Liu, X .G. Li, C. W Du, Materials Science \& Engineering A 685, 145-153, 2017

[2] S. Hiwatashi, A. V. Bael, P. V. Houtte, C. Teodosiu, Computational Materials Science 9, 274-284, 1997

[3] S. Hiwatashi, A. V. Bael, P. V. Houtte and C. Teodosiu, International Journal of Plasticity, 14, 7, 647-569, 1998

[4] R. Mesrar, S. Fromentin, R. Makkouk, M. Martiny and G. Ferron, International Journal of Plasticity, 14, s4-5, 391-411, 1998 
[5] M.S. Joo, D.W. Suh, J.H. Bae, H.K.D.H. Bhadeshia, Materials Science and Engineering A 546, 314-322, 2012

[6] M.S. Joo, D.-W. Suh, J.H. Bae, Sanchez Mourin, R. Petrov, L.A.I. Kestens, H.K.D.H. Bhadeshia, Materials Science and Engineering A 556, 601-606, 2012

[7] W. Chen, Q. Y. LIU, X. J. SUN, J. C. CAO, Journal of Iron and Steel Research, 22, 12, 53-58, 2010 (in Chinese)

[8] M. P. Butron-Guillen, J. J. Jonas , R. K. Ray, Acta- Metall Mater,42, 11, 3615-3627, 1994

[9] R. K. Ray, M. P. Butron-Guillen, J. J. Jonas, G. E. Ruddle, ISIJ International, 32, 2, 203-212, 1992

[10] G. J. Baczynski, J. J. Jonas, L. E. Collins, Metallurgical and materials transactions A, 30, 12, 3045-3054, 1998 